\title{
Comunicação
}

[Communication]

\section{Comportamento alimentar de paralarvas do polvo Octopus vulgaris Tipo II (Cuvier, 1797) alimentadas com artêmia enriquecida com microalgas e suplementada com DHA}

\author{
[Feeding behavior of octopus Octopus vulgaris Type II (Cuvier, 1797) paralarvae fed on Artemia enriched with \\ microalgae and supplemented with DHA] \\ P. Bastos ${ }^{1}$, G.C. Vieira ${ }^{1}$, I.M.M. dos Reis ${ }^{1}$, R.L. Costa ${ }^{2}$, G.R. Lopes $^{1}$ \\ ${ }^{1}$ Aluno de pós-graduação - Universidade Federal de Santa Catarina - Florianópolis, SC \\ ${ }^{2}$ Universidade Federal de Santa Catarina - Florianópolis, SC
}

As espécies do complexo Octopus cf. vulgaris (Cuvier, 1797) são consideradas potenciais para a diversificação da aquicultura marinha devido ao alto valor comercial, ao crescente demanda de consumo nos mercados asiático, europeu e latino-americano (Iglesias e Fuentes, 2014) e a características zootécnicas favoráveis ao cultivo. Em razão das recentes evidências de diferenças morfológicas e genéticas entre o Octopus vulgaris 'stricto sensu' (Mediterrâneo) e suas diferentes populações, o $O$. vulgaris 'do Brasil' está classificado como $O$. vulgaris Tipo II (Brasil), sua taxonomia está sendo reavaliada (Amor et al., 2017). O polvo O. vulgaris apresenta ciclo de vida curto, com taxas de crescimento de até $1 \mathrm{~kg} / \mathrm{mês}$, eficiente conversão alimentar, alta fecundidade, fácil adaptação ao cultivo, além de serem excelente fonte de proteína para consumo humano (Vaz-Pirez et al., 2004).

O principal gargalo para o cultivo comercial do $O$. vulgaris é a alta mortalidade nos primeiros dois meses da fase de larvicultura devido à qualidade nutricional das dietas ofertadas que não suprem as exigências nutricionais das paralarvas (Iglesias e Fuentes, 2014). Uma das grandes dificuldades é oferecer um alimento com baixo teor lipídico e rico em ácidos graxos poliinsaturados de cadeia longa (LCPUFA), em particular, os ácidos graxos eicosapentaenoico (EPA) e docosahexaenoico (DHA) em proporções adequadas (Navarro e Villanueva, 2003). No cenário atual, não há dietas formuladas balanceadas que promovam crescimento e sobrevivência adequados na fase de larvicultura do $O$. vulgaris, portanto o sucesso do cultivo depende da qualidade e da quantidade das presas vivas ofertadas como alimento.

As maiores taxas de crescimento e sobrevivência na larvicultura do $O$. vulgaris têm sido alcançadas com alimento natural, baseado em dieta mista de zoeas de caranguejo e artêmia (Iglesias et al., 2014). Contudo, a produção de zoeas de crustáceos em larga escala inviabiliza economicamente a transferência dessa tecnologia para o cultivo comercial de polvo. A utilização de artêmia como presa viva na larvicultura é importante pela viabilidade de produção em grande quantidade em laboratório e por ela ser facilmente aceita pelas paralarvas. Embora a artêmia possua inadequada composição de ácidos graxos (Navarro e Villanueva, 2003), apresenta alta relação proteína/lipídeos, podendo contribuir positivamente para o crescimento das paralarvas (Fuentes et al., 2011). Uma das principais práticas utilizadas para melhorar a composição nutricional das artêmias a serem ofertadas como primeira alimentação para as paralarvas é o enriquecimento com microalgas, tais como Tetraselmis suecica, Chaetoceros sp., Isocrysis galbana, Rhodomonas lens e Nannochloropsis sp. (Hamasaki e Takeuchi, 2000; Moxica et al., 2002; Seixas et al., 2010; Fuentes et al., 2011). Contudo, apenas Moxica et al. (2006) alcançaram a produção do $O$. vulgaris com ciclo completo em laboratório, utilizando artêmia enriquecida com microalgas como única dieta, e um polvo chegou à fase adulta.

Recebido em 9 de fevereiro de 2017

Aceito em 22 de junho de 2017 ,

E-mail: penelopebastos@gmail.com 
No Brasil, apesar do crescente interesse no cultivo de polvos, são poucos os resultados sobre larvicultura de $O$. vulgaris. As primeiras experiências permitiram obter paralarvas até 40 dias após eclosão (DAE), alimentadas com zoés de crustáceo, misidáceos, copépodos e artêmia enriquecida com DHA (Iglesias et al., 2007) e ainda há uma grande lacuna até obter indivíduos com qualidade na fase de assentamento. De fato, a larvicultura do $O$. vulgaris precisa ser explorada de forma mais eficiente, para viabilizar a produção comercial dessa espécie.

O presente trabalho relata o comportamento alimentar de paralarvas do Octopus vulgaris Tipo II alimentadas com Artemia sp. enriquecidas com as microalgas Isocrysis galbana e Pavlova lutheri e suplementadas com emulsão lipídica DHA.

O experimento foi realizado em sistema parcial de recirculação de água $(50 \%$ do volume por hora), com água do mar filtrada $(1 \mu \mathrm{m})$, esterilizada com ultravioleta (UV), constituído por três tanques circulares de 500L $(101,2 \mathrm{~cm}$ de diâmetro de fundo e $63,0 \mathrm{~cm}$ de altura) para o cultivo das paralarvas.

Os tanques de cultivo foram adaptados com uma entrada e duas saídas de água (Fig. 1). O centro de cada tanque continha um dreno, com abertura de $75 \mathrm{~mm}$ de diâmetro, que, por escoamento, descartava água pela saída inferior dos tanques. A lateral dos tanques possuiu uma saída acoplada a um tubo de polietileno revestido com tela de malha com abertura de $180 \mu \mathrm{m}$, para evitar a fuga das paralarvas (Fig. 1B). Nessa saída lateral, a água era bombeada para um filtro pressurizado de areia (filtro tipo piscina) e, em seguida, para filtros biológicos. Após o tratamento, a água era redistribuída novamente para os tanques de cultivo. O fluxo de água nos tanques de cultivo foi controlado por registros, gerando uma vazão de $0,5 \mathrm{~L} \cdot \mathrm{min}^{-1}$, o que forneceu uma renovação de água de $144 \% \cdot$ dia $^{-1}$. A aeração foi mantida constante, o oxigênio em $90 \%$ de saturação e o fotoperíodo natural, 12L:12E (período de luz entre sete horas e 19h). A limpeza diária dos tanques foi realizada por meio da retirada do tubo central (a) e do tubo externo (b), permitindo a drenagem central (Fig. 1B) e a renovação de $25 \%$ do volume total do sistema. A cada quatro dias, foi sifonado o fundo dos tanques.
Para estabelecer o sistema "água verde", foram introduzidas diariamente no sistema as microalgas Isocrysis galbana e Pavlova lutheri, ajustando-se a concentração total de 50000cel. $\mathrm{mL}^{-1}$. As paralarvas recém-eclodidas foram obtidas da desova de uma fêmea mantida em um tanque circular de 500L, em laboratório. Em seguida, as paralarvas foram cuidadosamente transferidas para as unidades experimentais. Foram transferidas 24000 paralarvas distribuídas ao acaso nos três tanques, até alcançar a densidade de 8000 paralarvas por tanque (16 paralarvas. $\left.\mathrm{L}^{-1}\right)$.

As paralarvas foram alimentadas diariamente com artêmia (Artemia High 5, INVE ${ }^{\circledR}$, Bélgica), na proporção de 0,1 artêmia.mL ${ }^{-1}$. A partir do dia zero (dia após a eclosão - DAE), foram ofertados náuplios de artêmia $(0,5$ a $0,6 \mathrm{~mm}) \mathrm{e}$, a partir do 8DAE, foram ofertados metanáuplios (1,0 a $4,0 \mathrm{~mm})$. As artêmias foram obtidas por meio da eclosão dos cistos de acordo com metodologia de Sorgeloos et al. (2001) e cultivadas em laboratório até a fase de metanáuplio.

Para obtenção de náuplios, diariamente os cistos de artêmia $(1,5 \mathrm{~g} / \mathrm{L})$ foram transferidos para tanques cilíndricos cônicos de $250 \mathrm{~L}$, com forte aeração e iluminação artificial, com água do mar $(1 \mu \mathrm{m})$ filtrada, esterilizada com UV, aquecida constantemente a $26^{\circ} \mathrm{C}$ e salinidade 35ups, mantidos por 24 horas até a eclosão. Após eclodirem os cistos, a aeração do tanque cilíndrico cônico foi desligada. Os náuplios de artêmia foram separados dos resíduos de incubação. Os cistos não eclodidos flutuaram para a parte superior do tanque e os náuplios recém-eclodidos se concentraram na parte inferior. Após 15 minutos, os náuplios foram evacuados cuidadosamente através da passagem inferior do tanque cilíndrico cônico, coletados com o auxílio de uma peneira de $120 \mu \mathrm{m}$ e destinados à alimentação de paralarvas. Náuplios recém-eclodidos não se alimentam por cerca de seis a oito horas devido a sua reserva energética (Benijts et al., 1976). Por esta razão, após o período de oito horas, parte dos náuplios recém-eclodidos foram cultivados em tanques cilíndricos cônicos de $250 \mathrm{~L}$, a uma densidade de até oito milhões de náuplios por litro, com aeração moderada, com água do mar $(1 \mu \mathrm{m})$ filtrada, esterilizada com UV, temperatura de $26^{\circ} \mathrm{C}$, salinidade $35 \mathrm{ups}$, e alimentados com uma dieta mista de microalgas Isocrysis galbana e 
Pavlova lutheri (1:1), na concentração de $10 \times 10^{4}$ células $\mathrm{mL}^{-1}$, enriquecidas com emulsão lipídica "DHA Selco ${ }^{\circledR}$ DC" (DHA, INVE, Bélgica), na proporção de $1 \mathrm{~g} / 500000$ artêmia, e mantidos nesse sistema por cinco a seis dias, até atingirem 4mm (metanáuplios).

Para contagem do número de artêmias e de paralarvas, foram retiradas alíquotas de $50 \mathrm{~mL}$ diariamente de cada tanque. Após a contagem, artêmias e paralarvas foram devolvidas aos respectivos tanques. Para observação da formação e do padrão de cromatóforos das paralarvas, foram coletados 10 indivíduos aleatoriamente de cada tanque a cada quatro dias. As paralarvas foram observadas em microscópio estereoscópico e fotografadas. A temperatura, a salinidade e o oxigênio da água nos tanques foram monitorados diariamente.

Com relação aos resultados dos parâmetros de qualidade de água, a temperatura e a salinidade, apresentaram valores (média \pm desvio-padrão) de $20,0 \pm 1,9^{\circ} \mathrm{C}$

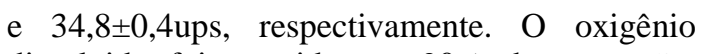
dissolvido foi mantido em $90 \%$ de saturação. Esses valores estão dentro dos níveis ótimos recomendados para garantir sobrevivência e o crescimento das paralarvas (Iglesias e Fuentes, 2014).

Quanto ao comportamento das paralarvas, foi observada natação ativa, alimentando-se na porção mais superficial da água, com boa aceitação da artêmia durante os primeiros 14DAE. Aos 15DAE, as paralarvas começaram a nadar verticalmente até a primeira metade da coluna d'água, permanecendo um pouco abaixo da superfície da água. A partir desse período, houve diminuição no consumo do alimento vivo e foram verificadas sobras de artêmias não consumidas nos tanques. Nos 18DAE, a taxa de sobrevivência das paralarvas, verificada por meio da contagem de cinco subamostras de $2 \mathrm{~L}$, foi de $80 \%$. Aos 20DAE, foi constatada mortalidade em massa das paralarvas.
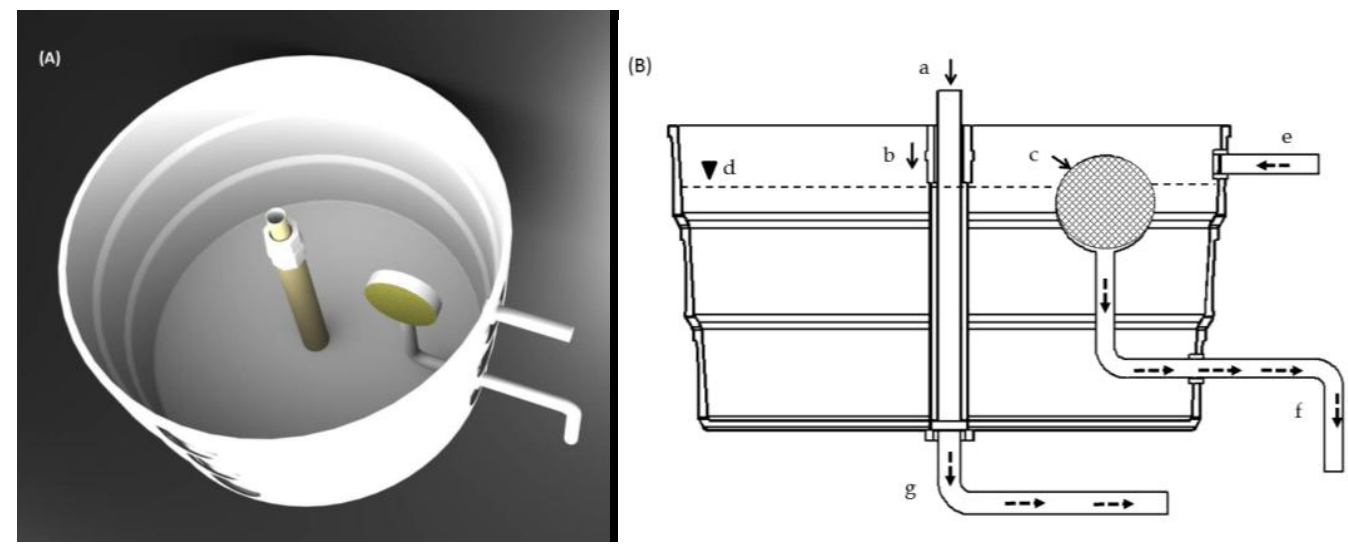

Figura 1. A) Vista em perspectiva do tanque de manutenção das paralarvas. B) Corte pleno AA do tanque de cultivo de paralarva. (a) Tubo de PVC de $75 \mathrm{~mm}$ para dreno central, (b) tubo de PVC de $100 \mathrm{~mm}$ como suporte ao tubo "a", (c) estrutura de PVC com tela de $180 \mu \mathrm{m}$, (d) nível de água, (e) entrada de água, (f) saída de água para recirculação, (g) saída de água para descarte.

No presente estudo, verificou-se que a natação vertical das paralarvas ocorreu concomitantemente às sobras de artêmia no tanque, o que provavelmente está relacionado com a baixa eficiência da interação presapredador. Na fase de larvicultura, o fluxo de água no tanque deve ser suficiente para permitir uma homogeneização adequada das presas, tornandoas disponíveis para serem capturadas pelas paralarvas (Vidal et al., 2002). Embora o fluxo de água tenha promovido boa distribuição das paralarvas na coluna d'água, possivelmente tenha sido insuficiente para promover uma boa homogeneização das paralarvas e das artêmias a partir da segunda semana de cultivo, mesmo com aeração moderada. O fato de a entrada e a saída da água estarem localizadas na parte superior do tanque também pode ter dificultado essa homogeneização ao longo da coluna d'água, inibindo a interação entre as paralarvas e as artêmias, o que pode ter levado às sobras de artêmias não consumidas nos tanques e à redução 
da alimentação das paralarvas. Esta pode ser uma das causas da mortalidade das paralarvas a partir dos 18DAE e da mortalidade em massa aos 20DAE, verificada poucos dias após serem constatadas as sobras de artêmias. Além disso, o excesso de alimento não consumido pode ter elevado a concentração de amônia e nitrito a níveis críticos para as paralarvas, prejudicando a qualidade da água.

Outro fator importante é o tamanho da presa em relação à paralarva. Iglesias e Fuentes (2014) recomendam uso de Artemia sp. 1,0-4,0mm a partir dos 15DAE porque a oferta de presas maiores a partir dessa fase aumenta significativamente a taxa de crescimento das paralarvas. Além disso, a exigência com relação ao tamanho da presa aumenta à medida que as paralarvas crescem e, notavelmente, quando está próximo da fase de assentamento, período associado a altos picos de mortalidade (Vidal et al., 2014). No presente relato, o tamanho de até $4 \mathrm{~mm}$ das artêmias pode ter sido insuficiente para estimular a predação pelas paralarvas a partir do 15DAE, o que provavelmente ocasionou as sobras de artêmias não consumidas no tanque a partir dos 14 dias de cultivo.

De forma geral, as paralarvas apresentaram boa formação dos tentáculos e ventosas, cromatóforos bem desenvolvidos, com padrão de coloração vermelho-escuro (Fig. 2). Poucas deformações foram constatadas nas ventosas, nos tentáculos ou no manto. Essas observações são importantes, pois demonstram que a altura dos tanques e o fluxo de água permitiram uma distribuição adequada das paralarvas na coluna d'água, sem que estas se chocassem contra a parede dos tanques ou a saída de água, contribuindo para o bom desenvolvimento paralarval.

A taxa de sobrevivência das paralarvas alcançadas no presente estudo são promissoras, sendo superiores aos obtidos por Seixas et al. (2010), que relataram $20 \%$ de sobrevivência aos 15DAE, utilizando Artemia sp. enriquecida com as microalgas I. galbana e Rhodomonas lens. Entretanto, as maiores taxas de sobrevivência foram obtidas por Moxica et al. (2006), que obtiveram $67 \%$ de sobrevivência após 30 DAE, $0,9 \%$ após 70 dias, três indivíduos aos três meses e um polvo adulto ao final do ciclo, em sistema de água verde com Nannochloropsis sp. e artêmias cultivadas com I. galbana e enriquecidas com Nannochloropsis sp.

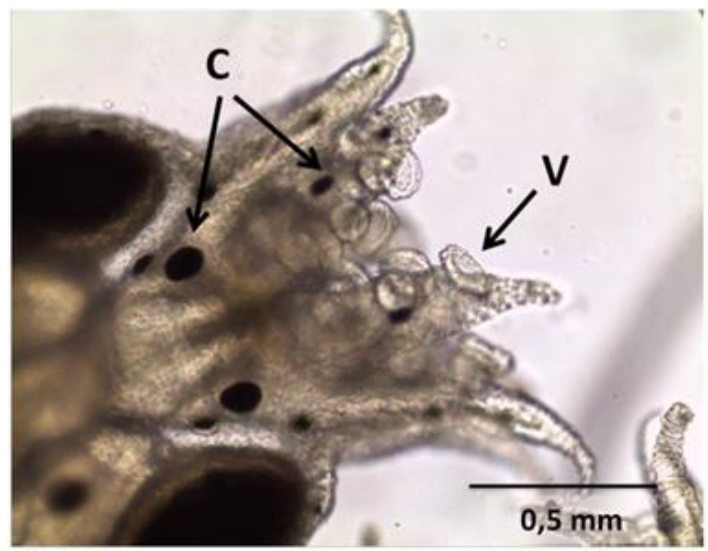

Figura 2. Paralarva de Octopus vulgaris Tipo II aos três dias de idade. $\mathrm{V}$ : ventosas, $\mathrm{C}$ : cromatóforos.

Hamasaki e Takeuchi (2000) alcançaram $88 \%$ de sobrevivência aos 24DAE, com o uso de Artemia sp. com e sem Nannochloropsis sp. A ação benéfica do uso de Nannochloropsis sp. combinada com I. galbana se deve à relação EPA:DHA, que atendem de forma mais eficiente as exigências em PUFA das paralarvas (Fuentes et al., 2011). Embora no presente estudo não tenha sido avaliada a composição nutricional da dieta, os resultados foram satisfatórios, demonstrados pela boa formação e comportamento ativo das paralarvas, associada à sobrevivência até $18 \mathrm{DAE}$. Avaliar o perfil de ácidos graxos dessa dieta e o ganho em peso das paralarvas poderá auxiliar a compreender se a mortalidade em massa está diretamente relacionada à qualidade e ao balanço lipídico da dieta.

Os resultados demonstram que é possível manter paralarvas de $O$. vulgaris Tipo II alimentadas com artêmia enriquecida com $I$. galbana e $P$. lutheri e suplementada com DHA pelo período de 18 dias. Estudos sobre a composição bioquímica e a ontogenia do sistema digestivo das paralarvas nos estágios iniciais do cultivo são essenciais para permitir maior conhecimento sobre a qualidade nutricional dessa dieta e sua aplicabilidade na larvicultura dessa espécie.

Palavras-chave: cefalópode, larvicultura, dieta, cultivo, aquicultura 


\begin{abstract}
The inexistence of nutritionally adequate diets in paralarval rearing is the main bottleneck for commercial production of the common octopus Octopus cf. vulgaris. We report the feeding behavior of $\mathrm{O}$. vulgaris Type II paralarvae fed on Artemia sp (0.1 individual. $\left.\mathrm{mL}^{-1}\right)$ nauplii enriched with microalgae Isocrysis galbana and Pavlova lutheri microalgae from 0 to 7 Day After Hatching $(D A H)$.; metanauplii enriched with microalgae and DHA SELCO ${ }^{\circledR}$ lipid emulsion from the $8 \mathrm{DAH}$. The paralarvae showed active swimming and predation by the $14 \mathrm{DAH}$, feeding in the most superficial portion of the water column. From the 15 DAH, the paralarvae remained near the bottom and there a decrease in the consumption of artemia was observed. The mortality observed from the $18 \mathrm{DAH}$ and mass mortality of paralarvae on $20 \mathrm{DAH}$ can be attributed mainly to the nutritional composition of the diet. Studies analyzing the biochemical composition and ontogeny of the digestive system during the early life stages should shed some light on the running for an appropriate feeding protocol to the paralarval rearing.
\end{abstract}

Keywords: cephalopod, larviculture, diet, cultivation, aquaculture

\section{AGRADECIMENTOS}

Ao Conselho Nacional de Pesquisa e Desenvolvimento (CNPq), pelo financiamento. Ao Dr. Jaime F. Ferreira e ao Dr. Francisco L. Squella, pela grande colaboração e apoio. À Isis B. Teixeira, pelo auxílio na formatação das imagens. Ao Lapmar (UFSC), pelo fornecimento das artêmias e do DHA.

\section{REFERÊNCIAS}

AMOR, M.; NORMAN, M.D.; A. ROURA, A. et al. Morphological assessment of the Octopus vulgaris species complex evaluated in the light of molecular-based phylogenetic inferences. Zool. Scrip., v.46(3), p. 275-288, 2017.

BENIJTS, F.; VANVOORDEN, E.; SORGELOOS, P. Changes in the biochemical composition of the early larval stages of the brine shrimp, Artemia salina L. In: EUROPEAN SYMPOSIUM ON MARINE BIOLOGY, 10., 1976, Wetteren. Proceendings.... Wetteren: Universa Press, 1976. v.1, p.1-9.

FUENTES, L.; SÁNCHEZ, F.J.; LAGO, M.J. et al. Growth and survival of Octopus vulgaris (Cuvier 1797) paralarvae fed on three Artêmia-based diets complemented with frozen fish flakes, crushed zooplankton and marine microalgae. Sci. Mar., v.75, p.771-777, 2011.

HAMASAKI, K.; TAKEUCHI, T. Effects of the addition of Nannochloropsis to the rearing water on survival and growth of planktonic larvae of Octopus vulgaris. Saibai Giken, v.28, p.65-68, 2000.

IGLESIAS, J.; FUENTES, L. Octopus vulgaris paralarval culture. In IGLESIAS, J.; FUENTES, L.; VILLANUEVA, R. (Eds.). Cephalopod culture. Amsterdam: Springer-Verlag, 2014. p.427-450.
IGLESIAS, J.; SÁNCHEZ, F.J.; BERSANO, J.G.F. et al. Rearing of Octopus vulgaris paralarvae: present status, bottlenecks and trends. Aquaculture, v.266, p.115, 2007.

MOXICA, C.; FUENTES, L.; HERNÁNDEZ, J. et al. Efecto de Nannochloropsis sp. en la supervivencia y crecimiento de paralarvas de pulpo Octopus vulgaris. In: FORO DOS RECURSOS MARIÑOS E DA ACUICULTURA DAS RÍAS GALLEGAS, 9., 2006, O Grove. Anales... O Grove: [Instituto de Acuicultura], 2006. p.255-261. (Resumo).

MOXICA, C.; LINARES, F.; OTERO, J.J. et al. Cultivo intensivo de pararlarvas de pulpo, Octopus vulgaris Cuvier, 1797, en tanques de 9 m3. Bol. Inst. Esp. Oceanogr., v.18, p.31-36, 2002.

NAVARRO, J.C.; VILLANUEVA, R. The fatty acid composition of Octopus vulgaris paralarvae reared with live and inert food: deviation from their natural fatty acid profile. Aquaculture, v.219, p.613-63, 2003.

SEIXAS, P.; REY-MÉNDEZ, M.; VALENTE, L.M.P. et al. High DHA content in Artemia is ineffective to improve Octopus vulgaris paralarvae rearing. Aquaculture, v.300, p.156-162, 2010.

SORGELOOS, P.; DHERT, P.; CANDREVA, P. Use of the brine shrimp, Artemia spp., in marine fish larviculture. Aquaculture, v.200, p.147-159, 2001.

VAZ-PIRES, P.; SEIXAS, P.; BARBOSA, A. Review: aquaculture potential of the common octopus Octopus vulgaris (Cuvier, 1797). Aquaculture, v.238, p.221-238, 2004.

VIDAL, E.A.G.; DIMARCO, F.P.; WORMUTH, J.H. et al. Optimizing rearing conditions of hatchling loliginid squid. Mar. Biol., v.140, p.117-127, 2002.

VIDAL, E.A.G.; VILLANUEVA, R.; ANDRADE, J.P.; GLEADALL, I.G. et al. Cephalopod culture: current status of main biological models and research priorities. Adv. Mar. Biol., v.67, p.1-98, 2014. 